\title{
Abnormal Region Extraction from MR Brain Images using Hybrid Approach
}

\author{
Nikhil Gala ${ }^{1}$ \\ Department of Electronics \& Telecommunication \\ Mukesh Patel School of Technology Management \& \\ Engineering, NMIMS University Mumbai, India
}

\author{
Dr. Kamalakar Desai ${ }^{2}$ \\ Academic and Technical Advisor \\ Guru Gobind Singh College of Engineering \& Research \\ Centre Nashik, India
}

\begin{abstract}
Automatic brain abnormality segmentation from magnetic resonance images is a key task that is performed by computer aided algorithm or manual extraction by a medical expert. The regions are often partitioned based on the similarities of intensities that persist in a particular region. MR brain image segmentation is a critical step that helps to identify the abnormal region. Accurate identification of this abnormal region helps the radiologist and surgeons in surgical process and research. Through this paper we present a hybrid approach of algorithms based on clustering approach like region and edge based algorithm involved in segmenting abnormal region from MR brain images. The method is an integration of region based (pillar K-means) and edge based (level set) segmentation algorithm that aims to segment the abnormal region precisely. Experimental results show that the proposed approach could attain segmentation efficiency of $89.2 \%$, mitigating the segmentation errors that were prevalent with region or edge based algorithms.
\end{abstract}

Keywords-Clustering algorithm; hybrid approach; MR brain image segmentation; level set; pillar k-means; segmentation errors

\section{INTRODUCTION}

Brain is considered as one of the vital and important part of human body which is made up of nerve cell called neurons and the supporting cells called Glial Cells which are meant to send and receive the messages to different parts of the body and thus control the body parts. The abnormality in the brain is an extra tissue that has grown in any part of the brain. In some cases the brain cells are multiplied in an uncontrolled manner from these regions. In general these tumors are classified as benign and malignant based on its growth and orientation. The benign type of tumor grows slowly and does not spread to other region. Malignant tumors grow at a much faster rate unlike benign tumors thus causing pressure on surrounding tissues that may lead to interference in body parts functionality. There are multiple imaging methods by which the abnormality is detected but Magnetic resonance imaging (MRI) has proved to be best in detecting brain abnormality this is due to its high contrast projection and high resolution. The human body parts are thus safe from getting direct exposure to multiple deteriorating radiations. This MR imaging of brain is considered in this work as it can produce the projections in three different view perspectives like sagittal, axial and coronal planes [1].

Multiple planar projections help to precisely locate and detect the abnormality or lesion regions. Hence, it can be stated that from discussed points it is apparent that T1-weighted MR brain imaging is more adequate for detection of abnormal regions which are segmented along with other components like edema and necrosis [2][3][18].

The traditional approach of analyzing MR tumor images by medical expert is often tedious and consumes a lot of time. Therefore it is suggested to have an automatic segmentation approach that can provide an equivalent performance to manual observation. Effective segmentation approach may locate and can be employed to measure the density and volume of the abnormality which is crucial in deciding the stage of severity.

Multiple approaches have been proposed so far by researchers and most of them belong to region based or edge based approaches. Edge based approaches rely and focus on the information that is present at the corners of the regions while the pixel or intensity based approach focuses on the internal variations of intensities. Despite of numerous algorithms for segmentation and extraction of abnormal regions MR brain image segmentation is still a challenging problem for researchers and the medical practitioners, this is because of the presence of multiple variations of regions in intensity and shapes. This paper focuses on presenting a hybrid approach of integrating the level set which is an edge based approach with pillar K-means algorithm which is a region based approach. The paper is structured as follows: Section 1 emphasizes on the need and necessity of the research and a basic introduction about MR brain imaging. Section 2 covers the literature on the related work that has been conducted earlier. Section 3 presents the background concepts that were used in the proposed approach. Section 4 presents the proposed approach with its outcome and comparisons with earlier approaches ending with the conclusions and discussion in Section 5.

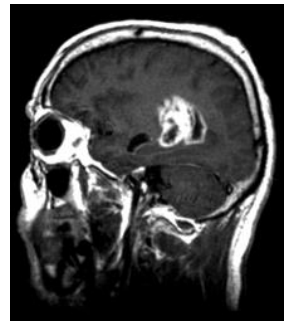

(a)

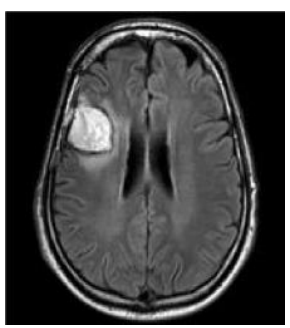

(b)

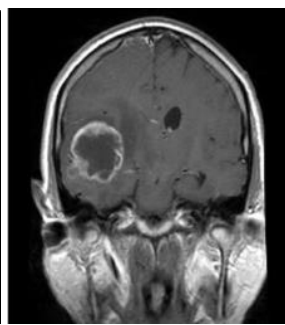

(c)
Fig. 1. MRI Brain Abnormality View in (a) Sagittal (b) Axial (c) Coronal. 


\section{RELATED WORK}

In [4] Maitra et al. has shown self-organizing maps based classification along with FCM (fuzzy C-means) clustering. The major contribution towards classification for MRI segmentation is done by Gibbs et al. [5], Zhu and Yan [6], Ho et al. [7]. Most extensively validated and appreciated system was presented by Clark et al. in [8] with two main components of this system being the Fuzzy C-Means.

A Multi class abnormal tissue i.e. brain tumor classification is proposed by $\mathrm{S}$ Dawood et al. in [9], using sparse coding and dictionary based learning. K-SVD algorithm is employed and the topological features are extracted to build the dictionary. The results of sparse are proven to be good than other methods. $3 \mathrm{D}$ image segmentation is proposed by Abbas and Farshad in [10] that aims to identify the image clusters and classify them. With this approach the processing time and memory utilization is reduced by $20 \%$ however the classification is performed using Jacquard's coefficient.

A combination of clustering and region growing approach is proposed by Hooda and Verma in [11], in which they combined the region growing approach with the benchmark fuzzy $\mathrm{C}$ - means and $\mathrm{K}$-means clustering approaches. Using this approach an accurate location and orientation of the abnormal tissue can be identified.

Segmentation with traditional Gradient Vector Flow (GVF) model was proposed by Tao wang et al. in [12] where they integrated the approach with traditional and BVF snake and magneto active contour model approaches. In this approach the GVF is applied for detecting the boundaries of the abnormal regions however this process is not suitable for $3 \mathrm{D}$ rendering hence interpolation is applied at the later stages.

A hybrid segmentation approach was proposed by $\mathrm{K}$. Verma et al. in [13] that aims to find exact contour of the abnormal regions. This approach includes watershed segmentation along with some edge operators integrated with morphological operations. This approach has been able to detect and analyze the size of the brain tumor region in the acquired MR brain images.

A combination of co-clustering approach with morphological operation for the extraction of tumor region was proposed by Satheesh et al. in [14]. Firstly, mathematical morphological operations were employed on T1-weighted MR images that intend to remove the non-brain regions and tissues including skull, fat and muscles; this process increases the efficiency of the segmentation algorithm. The skull and fat regions are often interfering with brain region during the process of segmentation resulting in inefficient segmentation. After the process of skull removal the later obtained brain region is subjected to co-clustering algorithm for segmentation of brain tumor.

Reyes et al. in [19], proposed an ROI based abnormal segmentation by integrating active contour models, clustering approach and some morphological operations. With this approach they could attain an efficiency of $88.2 \%$ than can be further increased.
Setyawan and others in [20] proposed a hybrid mechanism integrating clustering approach with morphological operations but could attain only $73.65 \%$ of accuracy.

Many of these methods have the limitations as in the case of edge and region based methods, so in order to overcome these deteriorations and accurately detect the abnormal region, a hybrid approach is proposed in this paper.

\section{BACKGROUND}

\section{A. Level-Set Approach using Non Re-Initialization}

Level set approach is one of the effective ways to implement active contours highly recommended to partition multiple regions from background. Many researchers have proposed several algorithms to deal with the problem of segmentation in computer vision however active contour models employed with level sets are more effective.

Active contours are employed through zero level set methods. This method can be realized as a function $\phi$ which is time dependent that varies according to the equation mentioned below

$\frac{\partial \phi}{\partial \mathrm{t}}+\mathrm{F}|\nabla \phi|=0$

Equation (1) is known as level set equation. In the above equation (1) the term " $F$ " is termed as speed function that relies on image data structure and $\phi$ which is the level set function. It is mandatory to ensure that the evolving level set function is very close to signed distance function such that a stable curve may attain during the process of implementing the level set approach. To own this criteria re-initialization of the function is not recommended however this may result to higher computation and numerical errors.

Let ' $I$ ' be an image, and ' $g$ ' is an edge indicator function which is defined by below equation (2)

$g=\frac{1}{1+\left|\nabla G_{\sigma^{* I}}\right|^{2}}$

Where $\mathrm{G}_{\sigma}$ is the Gaussian kernel with standard deviation $\sigma$.

The external energy of the function $\phi(x, y)$ is defined as

$\mathcal{E}_{g, \lambda, v}(\phi)=\lambda \mathcal{L}_{g}(\phi)+v \mathcal{A}_{g}(\phi)$

Where $\lambda>0$ and $v$ are constants.

The terms $\mathcal{L}_{g}(\phi)$ and $\mathcal{A}_{g}(\phi)$ are given by the below equations

$\mathcal{L}_{g}(\phi)=\int_{\Omega} g \delta(\phi)|\nabla \phi| d x d y$

$\mathcal{A}_{g}(\phi)=\int_{\Omega} g H(-\phi) d x d y$

Where $\delta$ is the univariate Dirac function, and $\mathrm{H}$ is the Heaviside function.

The total energy function is defined as

$\mathcal{E}(\phi)=\mu \mathcal{P}(\phi)+\mathcal{E}_{g, \lambda, v}(\phi)$ 
The term $\mathcal{P}(\phi)$ is called the internal energy function that penalizes the deviation of the function from the external energy and signed distance function. This inherits to drive the advancement of the zero level set towards region boundaries [15].

The evolution equation of this level set function is defined as

$\frac{\partial \phi}{\partial t}=\mu\left[\Delta \phi-\operatorname{div}\left(\frac{\nabla \phi}{|\nabla \phi|}\right)\right]+\lambda \delta(\phi) \operatorname{div}\left(g \frac{\nabla \phi}{|\nabla \phi|}\right)+v g \delta(\phi)$

\section{B. Segmentation using Pillar K-Means}

Let us assume that there exists a data $X=\left\{x_{i} \mid i=1, \ldots, n\right\}$ and ' $\mathrm{k}$ ' being the clusters and $\mathrm{C}=\left\{\mathrm{c}_{\mathrm{i}} \mid \mathrm{i}=1, \ldots, \mathrm{k}\right\}$ where $\mathrm{C}$ represents the original position of centroids. Let there exists a subset SX $\subseteq \mathrm{X}$ which identifies the term $\mathrm{X}$ and selected in the subsequent process. Let the distance metric between the elements is termed as $\mathrm{DM}=\{\mathrm{xi} \mid \mathrm{i}=1, \ldots, \mathrm{n}\}$ and the accumulated distance is termed as $D=\{x i \mid i=1, \ldots, n\}$ that is calculated after each iteration. The mean of $X$ is denoted as ' $m$ ' $[16]$.

The steps of the proposed algorithm approach are described as below

- At first initialize $\mathrm{C}=\varnothing, \mathrm{SX}=\varnothing$, and $\mathrm{DM}=[$ ]

- Calculate the mean (m) and also calculate the

- distance D from X and ' $m$ '

- Initialize the number of neighbors $\operatorname{nmin}=\alpha . \mathrm{n} / \mathrm{k}$

- The maximum of distance is termed as dmax

- $\quad$ Mark the neighborhood boundary as nbdis $=\beta$. dmax

- Initialize the iteration $\mathrm{i}=0$ and determine the ith initial centroid.

- Update the distance metrics $\mathrm{DM}=\mathrm{DM}+\mathrm{D}$

- Select the maximum of distance metric and mark it as ж

- Update the subset with the marked $\%$ as $\mathrm{SX}=\mathrm{SX}$ U ж

- Now calculate the distance between X set to ж

- The number of points fulfilling $\mathrm{D} \leq$ nbdis are termed as no

- $\quad$ Reset $\operatorname{DM}(ж)=0$

- If no $<$ nmin, go to step 8

- Assign $\mathrm{D}(\mathrm{SX})=0$

- $\mathrm{C}=\mathrm{C}$ U

- $\mathrm{i}=\mathrm{i}+1$

- If $\mathrm{i} \leq \mathrm{k}$, we need to update the distance matrix as per step 7

- Finish with $\mathrm{C}$ being the solution as per the optimized initial centroids.

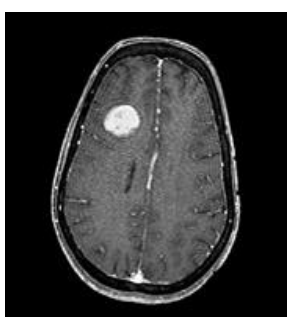

(a)

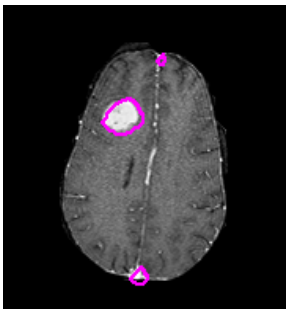

(d)

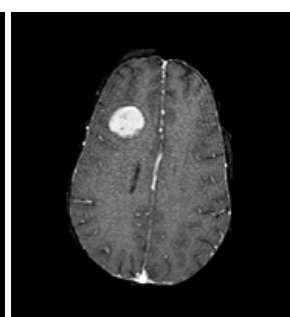

(b)

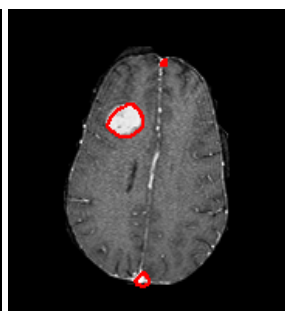

(e)

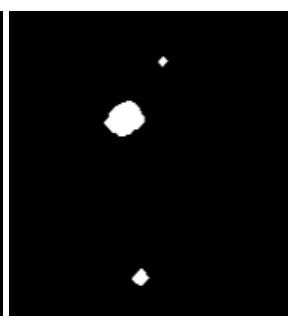

(c)

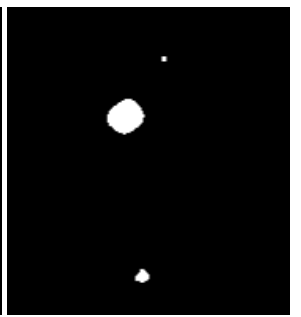

(f)
Fig. 2. Output at Various Stages (a) Original Image (b) Skull Removed (PreProcessed Image) (c) Pillar K-Means Segmented Image (d) Initial Contour for Level Set (e) Final Contour Obtained after 500 Iterations (f) Final Segmented Image.

\section{PROPOSED APPROACH}

The proposed approach is an integration of edge and region based algorithm. In this analysis T1-weighted MR image is subjected to pre-processing where the skull and fat regions are removed. Thus, obtained image is directed to pillar K-means clustering algorithm which is mentioned in the above section. The output of the clustering may contain segmentation blobs and it was observed that the over segmentation ratio is more. To mitigate this effect the boundary of the clustered abnormal region is given as an initial contour for the level set approach. In this present paper the skull removal approach mentioned by Satheesh et al. in [17] is utilized.

The step by step process is depicted in the below Figure 2 . The original image is preprocessed which helps in accurate detection and extraction of abnormal region.

In order to evaluate the performance obtained for the proposed approach, the algorithm is tested with 20 patients data recorded with 1.5 Philips achieva device. As it is known that the abnormal regions are clearly visible in few of the slices, two or three slices are considered for each patient and few of the results are tabulated below.

Performance evaluation is carried out and tabulated for 8 MR images of different patients. The same MR images are used for all the 3 approaches i.e. Pillar K-means, proposed approach (Pillar + level set) and Pillar + GVF approach for comparative analysis.

Figure 3 shows the segmented output of the applied algorithms and proposed approach along with manual segmented image by expert radiologist. Table 1 shows the performance of Pillar K-means. Table 2 shows the proposed approach (Pillar + level set) output. Results are tabulated for Pillar + GVF in Table 3. 


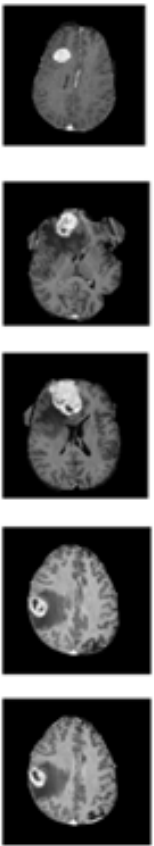

(a)
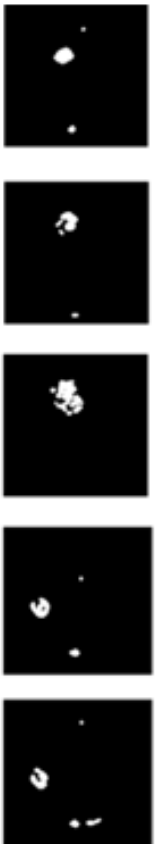

(b)
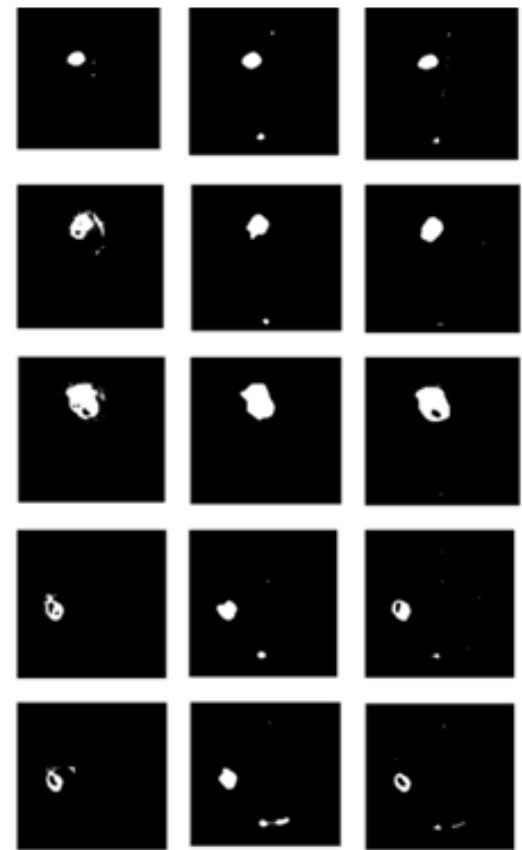

(c)

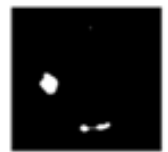

(d)
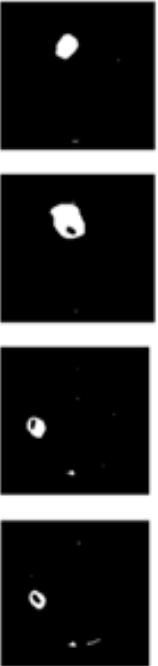

(e)

Fig. 3. (a) This Column Represents Original Pre-Processed Images (b) this Column Represents the Segmented Outputs with Pillar K-means Algorithm (c) this Column Represents the GVF+ Pillar Approach (d) this Column Represent the Segmented Output with Proposed Hybrid Approach (e) this Column Represents the Manual Segmented Images by Experts.

TABLE I. READINGS OBTAINED FOR METRIC ANALYSIS USING PILlAR KMEANS

\begin{tabular}{|c|c|c|c|c|c|}
\hline Image & \multicolumn{5}{|c|}{ Pillar K-means } \\
\hline & SI & CDR & OSE & USE & TSE \\
\hline & 0.855 & 0.982 & 0.315 & 0.017 & 0.33 \\
\hline & 0.725 & 0.649 & 0.139 & 0.305 & 0.409 \\
\hline & 0.805 & 0.716 & 0.063 & 0.283 & 0.346 \\
\hline & 0.8 & 0.877 & 0.313 & 0.122 & 0.436 \\
\hline & 0.685 & 0.916 & 0.759 & 0.083 & 0.842 \\
\hline & 0.613 & 0.476 & 0.52 & 0.07 & 0.6 \\
\hline & 0.68 & 0.58 & 0.42 & 0.098 & 0.52 \\
\hline & 0.59 & 0.31 & 0.52 & 0.06 & 0.58 \\
\hline AVG & 0.719 & 0.688 & 0.381 & 0.129 & 0.507 \\
\hline
\end{tabular}

TABLE II. READING OBTAINED FOR METRIC ANALYSIS USING PILLAR AND LEVEL-SET APPROACH

\begin{tabular}{|c|c|c|c|c|c|}
\hline \multirow[b]{2}{*}{ Image } & \multicolumn{5}{|c|}{ Hybrid (Pillar + level-set) } \\
\hline & SI & CDR & OSE & USE & TSE \\
\hline & 0.865 & 0.945 & 0.22 & 0.05 & 0.28 \\
\hline & 0.84 & 0.832 & 0.141 & 0.165 & 0.317 \\
\hline & 0.86 & 0.862 & 0.143 & 0.137 & 0.28 \\
\hline & 0.812 & 0.887 & 0.299 & 0.112 & 0.413 \\
\hline & 0.673 & 0.935 & 0.842 & 0.064 & 0.907 \\
\hline & 0.645 & 0.52 & 0.48 & 0.092 & 0.57 \\
\hline & 0.79 & 0.69 & 0.3 & 0.069 & 0.36 \\
\hline & 0.68 & 0.65 & 0.44 & 0.088 & 0.48 \\
\hline AVG & 0.77 & 0.792 & 0.358 & 0.09 & 0.454 \\
\hline
\end{tabular}

TABLE III. READING OBTAINED FOR METRIC ANALYSIS USING PILLAR AND GVF APPROACH

\begin{tabular}{|c|c|c|c|c|c|}
\hline \multirow[t]{2}{*}{ Image } & \multicolumn{5}{|c|}{ Hybrid (Pillar + GVF) } \\
\hline & SI & CDR & OSE & USE & TSE \\
\hline & 0.862 & 0.833 & 0.07 & 0.16 & 0.241 \\
\hline & 0.777 & 0.917 & 0.442 & 0.082 & 0.525 \\
\hline & 0.877 & 0.927 & 0.138 & 0.072 & 0.211 \\
\hline & 0.807 & 0.803 & 0.186 & 0.196 & 0.382 \\
\hline & 0.717 & 0.778 & 0.278 & 0.221 & 0.5 \\
\hline & 0.62 & 0.51 & 0.56 & 0.18 & 0.74 \\
\hline & 0.72 & 0.68 & 0.39 & 0.16 & 0.55 \\
\hline & 0.66 & 0.50 & 0.49 & 0.027 & 0.52 \\
\hline AVG & 0.758 & 0.743 & 0.312 & 0.137 & 0.458 \\
\hline
\end{tabular}


The proposed method is compared with Pillar K-means [16], Pillar + GVF approach and the metrical analysis is calculated with respected to manual segmented images by experts. Multiple metrics like segmentation efficiency/ Similarity Index (SE/SI), correct detection ratio (CDR), over segmentation error (OSE), under segmentation error (USE) and total segmentation error (TSE) that were mentioned in [17] were adopted for evaluating the performance of this segmentation approach. Table 4 below shows the comparative analysis of the proposed approach using these metrics. Bold values indicate better performance. Figure 4, Figure 5 and Figure 6 helps to understand that the proposed approach is more efficient in detection of the abnormal region and further reduces segmentation errors. As seen in Figure 7, the proposed approach has given better performance when SI, CDR, and TSE is compared.

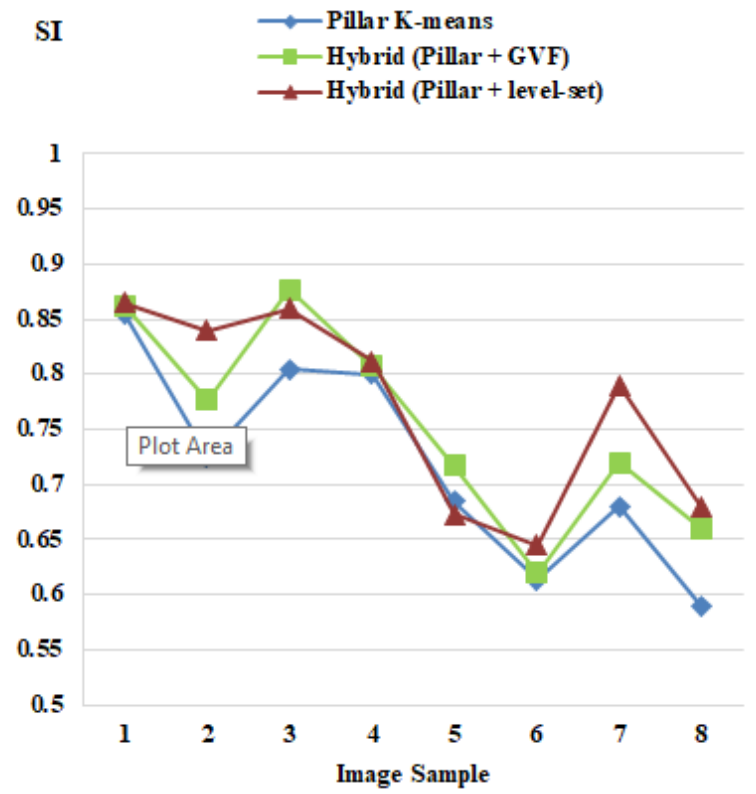

Fig. 4. Graph Representing Similarity Index Comparison.
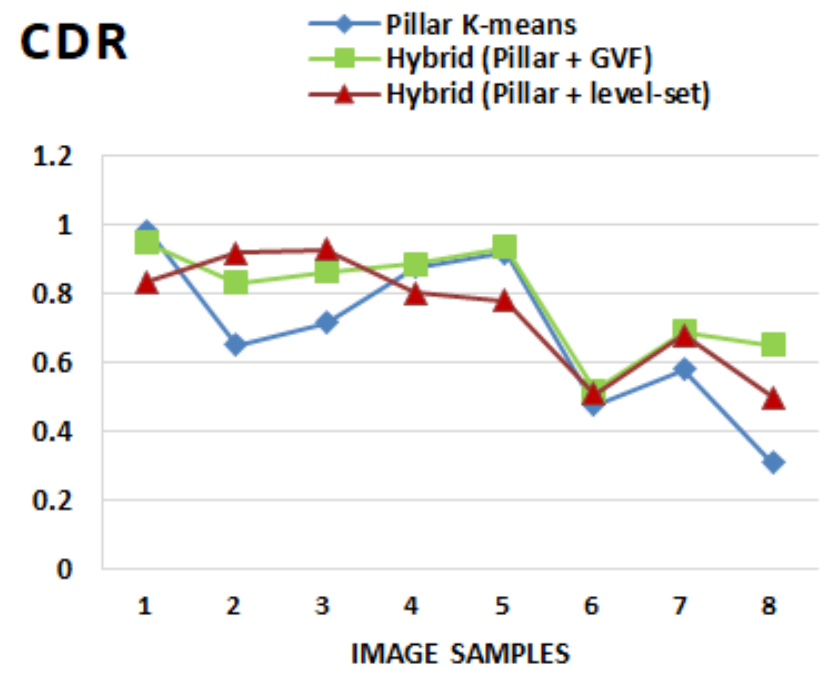

Fig. 5. CDR of Pillar K-Mean, Pillar+GVF and Proposed Approach.
TABLE IV. COMPARATIVE PERFORMANCE OF PROPOSED APPROACH

\begin{tabular}{|l|l|l|l|l|l|}
\hline Approach & SI & CDR & OSE & USE & TSE \\
\hline Pillar K-means & 0.719 & 0.688 & 0.381 & 0.129 & 0.507 \\
\hline Pillar + level-set & $\mathbf{0 . 7 7}$ & $\mathbf{0 . 7 9 2}$ & 0.358 & $\mathbf{0 . 0 9}$ & $\mathbf{0 . 4 5 4}$ \\
\hline Pillar + GVF & 0.758 & 0.743 & $\mathbf{0 . 3 1 2}$ & 0.137 & 0.458 \\
\hline
\end{tabular}
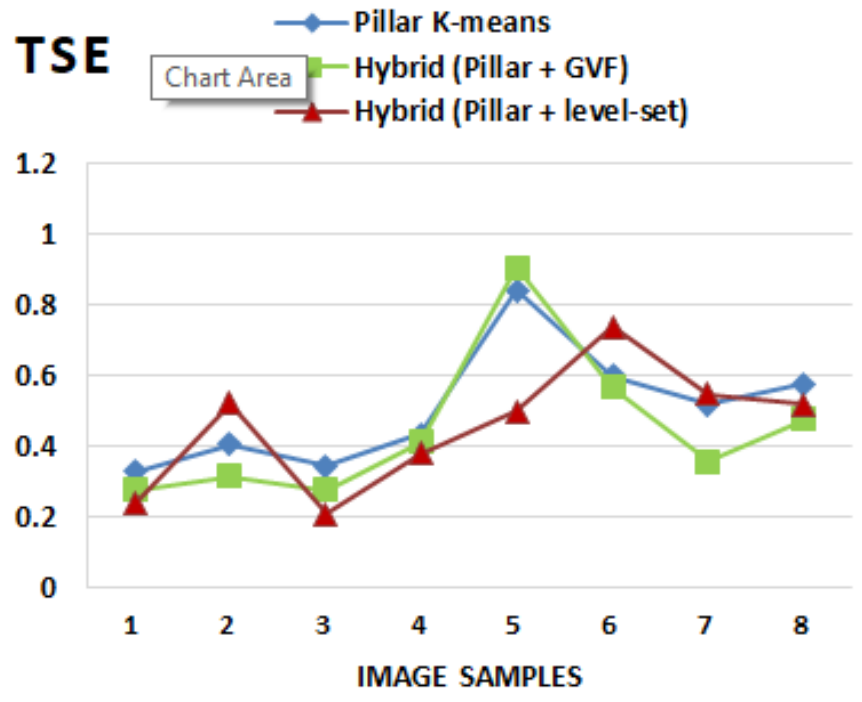

Fig. 6. Comparison of Total Segmentation Error.

$$
\begin{aligned}
& - \text { Pillar K-means } \\
& - \text { Hybrid (Pillar + level-set) } \\
& - \text { Hybrid (Pillar + GVF) }
\end{aligned}
$$

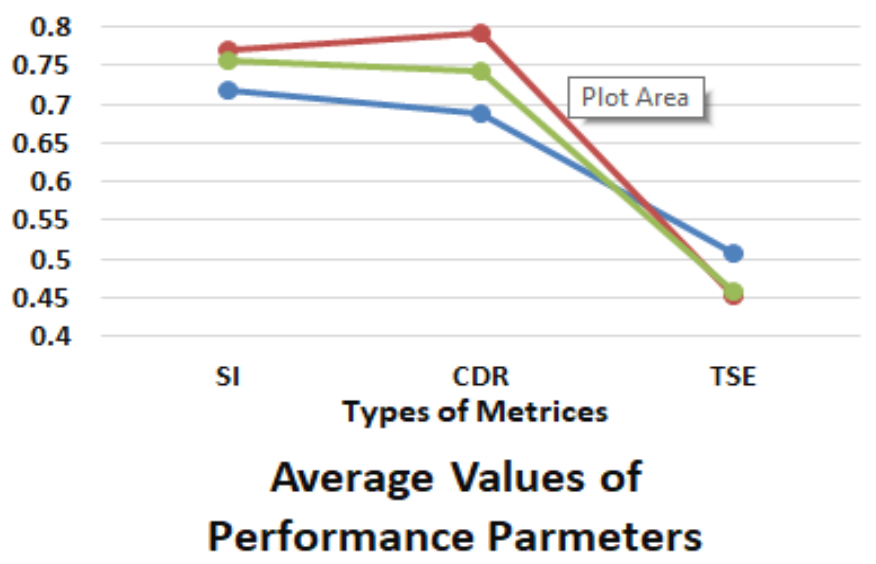

Fig. 7. Comparative Analysis of Proposed Approach.

\section{CONCLUSION}

This work presents a new low complex hybrid segmentation approach that incorporates pillar K-means and a level set method proposed. The method is able to efficiently segment the abnormal region from the input pre-processed images. The performance achieved from the proposed approach is compared with the pillar K-means and found that in all aspects it is yielding better results. In precise when 
compared with integrated GVF approach with pillar K-means the method has gained $2 \%$ more similarity and attained $5 \%$ more correct detection. However, the method has over segmentation error larger than Pillar + GVF approach by $4 \%$ that has to be mitigated. Finally, it can be concluded that the proposed approach has been able to reach the objectives of this work in minimizing the segmentation errors that were occurring with traditional clustering approaches.

\section{REFERENCES}

[1] American Society of Neuroradiology. "ACR-ASNR Practice Guideline for the Performance and Interpretation of Magnetic Resonance Imaging (MRI) of the Brain" -2013

[2] Galloway, RL Jr, "Introduction and Historical Perspectives on ImageGuided Surgery ", In Golby, AJ, Image-Guided Neurosurgery, Amsterdam: Elsevier. pp. 3-4, 2015

[3] TSE, VCK; Kalani, MYS; Adler, JR, "Techniques of Stereotactic Localization". In Chin, LS; Regine, WF. Principles and Practice of Stereotactic Radio surgery. New York: Springer. pg.no 28,2015

[4] M. Maitra, A. Chatterjee,"Hybrid multi resolution Slantlet transform and fuzzy c-means clustering approach for normal-pathological brain MR image segregation", Medical Engineering and Physics, Elsevier Publishers, 2007.

[5] Gibbs, P., Buckley, D., Blanckb, S., and Horsman, A.," Tumor volume determination from MR images by morphological segmentation", Physics in Medicine and Biology, 41:2437-2446, 1996.

[6] Zhu, Y. and Yan, H. "Computerized tumor boundary detection using a Hopfield neural network" IEEE Transactions on Medical Imaging, 16:55-67, 1997.

[7] Ho, S., Bullitt, E., and Gerig, G. "Level set evolution with region competition: automatic 3D segmentation of brain tumors", In 16th International Conference on Pattern Recognition, pages 532-535,2002.

[8] Clark, M., Hall, L., Goldgof, D., Velthuizen, R., Murtagh, F., and Silbiger, M., "Automatic tumor segmentation using knowledge- based techniques. IEEE Transactions on Medical Imaging, 17:238-251,1998

[9] Al-Shaikhli, S. D. S., Yang, M. Y., \& Rosenhahn, B. "Brain tumor classification using sparse coding and dictionary learning", In 2014 IEEE International Conference on Image Processing (ICIP) (pp. 2774-2778). IEEE-2014

[10] Abbasi, S, Pour, F. T, "A hybrid approach for detection of brain tumor in MRI images", In Biomedical Engineering (ICBME), 2014 21th Iranian Conference on (pp. 269-274). IEEE, 2014
[11] Hooda, H, Verma, O. P., \& Singhal, T., "Brain tumor segmentation: A performance analysis using K-Means, Fuzzy C-Means and Region growing algorithm". In Advanced Communication Control and Computing Technologies (ICACCCT), 2014 International Conference on (pp. 1621-1626), 2014

[12] T. Wang, I. Cheng and A. Basu, "Fluid Vector Flow and Applications in Brain Tumor Segmentation," in IEEE Transactions on Biomedical Engineering, vol. 56, no. 3, pp. 781-789, March 2009.

[13] Verma, Kimmi, and Shabana Urooj. "Effective evaluation of tumor region in brain MR images using hybrid segmentation." 2014 International Conference on Computing for Sustainable Global Development (INDIACom), 2014

[14] S.Satheesh, Dr.K.V.S.V.R Prasad, Dr.K.Jitender Reddy, "Tumor Extraction And Volume Estimation For T1-Weighted Magnetic Resonance Brain Images", Global Journal of Computer Science and Technology Neural \& Artificial Intelligence, Volume 12 Issue 12 Version 1.0 Year 2012

[15] Chunming Li; Chenyang Xu; Changfeng Gui; Fox, M.D., "Level set evolution without re-initialization: a new variational formulation," Computer Vision and Pattern Recognition, 2005. CVPR 2005.IEEE Computer Society Conference on , vol.1, no., pp.430,436 vol. 1, 20-25 June 2005

[16] Ali Ridho Barakbah ; Yasushi Kiyoki, A pillar algorithm for K-means optimization by distance maximization for initial centroid designation, Computational Intelligence and Data Mining, 2009.

[17] S. Satheesh, R. T. Santosh Kumar, K.V.S.V.R Prasad and K. Jitender Reddy, "Skull removal of noisy magnetic resonance brain images using Contourlet transform and morphological operations," Proceedings of 2011 International Conference on Computer Science and Network Technology, Harbin, 2011, pp. 2627-2631.

[18] Nolen-Hoeksema, Susan. Abnormal Psychology (Sixth edn). New York, NY: McGraw-Hill Education. p. 67,-2014

[19] A. M. de los Reyes, M. Elena Buemi, M. N. Alemán and C. Suárez, "Development of a graphic interface for the three-dimensional semiautomatic glioblastoma segmentation based on magnetic resonance images," 2018 Congreso Argentino de Ciencias de la Informática y Desarrollos de Investigación (CACIDI), Ciudad Autónoma de Buenos Aires, Argentina, 2018, pp. 1-6.

[20] R. Setyawan, M. A. Almahfud, C. A. Sari, D. R. I. M. Setiadi and E. H. Rachmawanto, "MRI Image Segmentation using Morphological Enhancement and Noise Removal based on Fuzzy C-means," 2018 5th International Conference on Information Technology, Computer, and Electrical Engineering (ICITACEE), Semarang, Indonesia, 2018, pp. 99104. 